\title{
A retrospective review of the Pediatric Development Clinic implementation: a model to improve medical, nutritional and developmental outcomes of at-risk under- five children in rural Rwanda
}

Eric Ngabireyimana ${ }^{1 \dagger}$, Christine Mutaganzwa ${ }^{2,7^{*}}$, Catherine M. Kirk ${ }^{2}$, Ann C. Miller ${ }^{5}$, Kim Wilson ${ }^{4,5}$,

Evodia Dushimimana ${ }^{6}$, Olivier Bigirumwami ${ }^{1}$, Evelyne S. Mukakabano ${ }^{1}$, Fulgence Nkikabahizi ${ }^{1}$ and Hema Magge ${ }^{2,3,4}$

\begin{abstract}
Background: As more high-risk newborns survive the neonatal period, they remain at significant medical, nutritional, and developmental risk. However, no follow-up system for early intervention exists in most developing countries. In 2014, a novel Pediatric Development Clinic (PDC) was implemented to provide comprehensive followup to at-risk under-five children, led by nurses and social workers in a district hospital and surrounding health centers in rural Rwanda.

Methods: At each PDC visit, children undergo clinical/nutritional assessment and caregivers participate in counseling sessions. Social assessments identify families needing additional social support. Developmental assessment is completed using Ages and Stages Questionnaires. A retrospective medical record review was conducted to evaluate the first 24 months of PDC implementation for patients enrolled between April 2014December 2015 in rural Rwanda. Demographic and clinical characteristics of patients and their caregivers were described using frequencies and proportions. Completion of different core components of PDC visits were compared overtime using Fisher's Exact test and $p$-values calculated using trend analysis.
\end{abstract}

Results: 426 patients enrolled at 5 PDC sites. 54\% were female, 44\% were neonates and 35\% were under 6 months at enrollment. Most frequent referral reasons were prematurity/low birth weight (63\%) and hypoxic-ischemic encephalopathy (34\%). In 24 months, 2787 PDC visits were conducted. Nurses consistently completed anthropometric measurements (age, weight, height) at all visits. Some visit components were inconsistently recorded, including adjusted age $(p=0.003)$, interval growth, danger sign assessment, and feeding difficulties $(p<0.001)$. Completion of other visit components, such as child development counseling and play/stimulation activities, were low but improved with time $(p<0.001)$.

(Continued on next page)

\footnotetext{
* Correspondence: mutaganzwa@gmail.com

${ }^{\dagger}$ Equal contributors

2Department of Pediatrics, Partners In Health/Inshuti Mu Buzima, Rwinkwavu,

Rwanda

7P.O. Box 3432, Kigali, Rwanda

Full list of author information is available at the end of the article
} 
(Continued from previous page)

Conclusions: It is feasible to implement PDCs with non-specialized providers in rural settings as we were able to enroll a diverse group of high-risk infants. We are seeing an improvement in services offered at PDCs over time and continuous quality improvement efforts are underway to strengthen current gaps. Future studies looking at the outcomes of the children benefiting from the PDC program are underway.

Keywords: High-risk newborns, survival, early childhood development, primary care, sub-Saharan Africa

\section{Background}

Global advances have been made over the past decade to improve neonatal care and the burden of neonatal mortality and morbidity in low and middle-income countries [1] with prematurity as the leading cause of death among children under 5 years of age [2]. Ongoing efforts to eliminate preventable neonatal deaths globally must also be accompanied by efforts to ensure children not only survive but also thrive [3]. The first 3 years of life are a critical period of brain development [4]; interventions during this early period can improve growth and development [5] which impact long-term outcomes including educational attainment and productivity later in life $[6,7]$. Certain populations of children are at increased risk of developmental delay and growth faltering, such as those born preterm and low birth weight, or with hypoxic-ischemic encephalopathy (HIE), congenital malformations, genetic syndromes, or birth injuries. It is known that these high-risk infants can have a range of challenges such as vision and hearing impairment, speech and motor delays, and poor nutrition, among others [8-11]. In high-income countries, longitudinal follow-up of these children is integrated into routine pediatric care and is typically conducted by specialists [12]. In sub-Saharan Africa, there are very few interventions to support early childhood development and the majority are generalized, community-based programs $[13,14]$ that do not meet the unique needs of high-risk infants. The use of an intensive home-visiting model has shown promise for improving the development of children with HIE in low and middle income countries [15]. However, to our knowledge there are no early childhood development interventions integrated into routine primary care for at-risk children in low-income countries without access to pediatricians and subspecialists, especially in sub-Saharan Africa.

Rwanda has made tremendous improvements in reducing under-five mortality [16], and expanded access to neonatal care through neonatal units at district hospitals and essential neonatal care at health centers. However, there is no formal follow-up system for children after being discharged from hospital neonatal units, especially in rural areas where there are no specialists; these children remain at increased risk for mortality and morbidities into childhood [17]. "A follow-up of preterm and low birth weight children discharged from Rwinkwavu District Hospital neonatal unit between October 2011 and October 2013 showed rates of chronic and acute malnutrition that were double and triple national prevalence rates, respectively, and that caregivers frequently reported feeding difficulties. Furthermore, two-thirds of the children had an abnormal developmental screening (Kirk, et al., unpublished data)".

In Rwanda, screening for malnutrition in infants and young children is conducted at the community level by community health workers [18] and early childhood development programs have been piloted in selected communities $[19,20]$. As in many other sub-Saharan African countries, a barrier to delivering specialized follow-up is the limited availability of pediatricians outside of higherlevel referral facilities [21], and other specialists such as developmental pediatricians, neurologists, and physical, speech and occupational therapists. Task-shifting has been used as an approach across low-resource settings to overcome the gap between the availability of specialized providers and service needs [22]. However, this approach has not been explored for pediatric follow-up care among high-risk infants.

Since April 2014 the Ministry of Health, in collaboration with Partners In Health/Inshuti Mu Buzima and UNICEF, has been implementing the Pediatric Development Clinic (PDC) to serve neonates discharged from a rural hospital neonatal unit and other high-risk children under-five in need of follow-up. The PDC provides medical, nutritional and developmentally supportive programs to allow for early identification of complications and intervention to support children to reach their full potential. We hope that this study will provide a pragmatic approach to implementing a feasible clinic model for comprehensive medical, nutritional, developmental support for high-risk infants until age 5 years in resource-limited settings without access to complex care services and medical specialists.

\section{Methods}

\section{Study aim and design}

This study aims to provide a description of the PDC intervention's first 2 years of implementation using a retrospective electronic medical record review of routinely-collected data. The study population includes 
all children referred into PDC from April 2014-December 2015. In order to ensure all referred children completed at least one visit following their referral date, we analyzed all of the visits for these children in the first 2 years of clinic implementation (April 2014-March 2016). We hypothesize that as the program matures, over time we will see greater adherence to clinic procedures and increase in documentation of appropriate clinical and developmental assessment and follow-up.

\section{Study intervention}

This study was conducted in the Rwinkwavu District Hospital catchment area in Southern Kayonza District in rural Rwanda. Rwinkwavu District Hospital is a public Ministry of Health facility supported by Partners In Health/Inshuti $\mathrm{Mu}$ Buzima since 2005. Southern Kayonza serves a population of approximately 188,363 [23] with one district hospital and eight health centers. Rwinkwavu District Hospital has provided neonatal care since 2010 and was among the first district hospitals to initiate specialized neonatal care services [24]. The first PDC was opened in April 2014 at Rwinkwavu District Hospital to provide medical, nutritional and developmental follow-up for high-risk children discharged from the neonatal unit (see Fig. 1). Four months later, in August 2014, PDC was expanded to two health centers and thereafter to two more in June 2015.

The PDC serves children with prematurity, birth weight under $2000 \mathrm{~g}$, HIE, hydrocephalus, cleft lip and palate, trisomy 21 , or other developmental delays. In April 2015, additional referral criteria - post-central nervous system infections (meningitis and cerebral malaria) - were added, and in February 2016 (after the inclusion period for this study), children diagnosed with severe acute malnutrition requiring hospitalization began being referred to PDC after discharge from the hospital. Children are referred to PDC using standardized referral criteria and a form completed from Rwinkwavu District Hospital neonatal unit, maternity ward, pediatric ward or emergency department when a child meeting referral

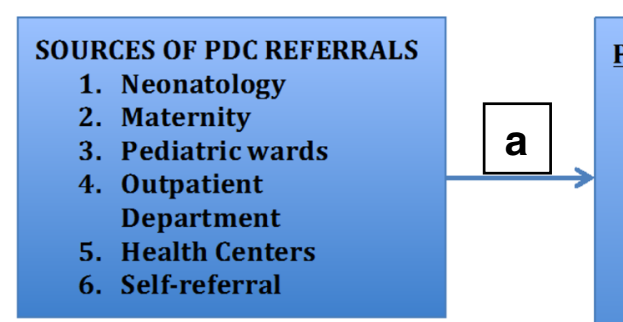

PDC clinic (Target population)

1. PT/LBW

2. HIE

3. Hydrocephalus

4. Trisomy 21

5. Cleft lip/palate

6. Post-CNS infections

7. SAM requiring hospitalization

8. Developmental delay

9. Others

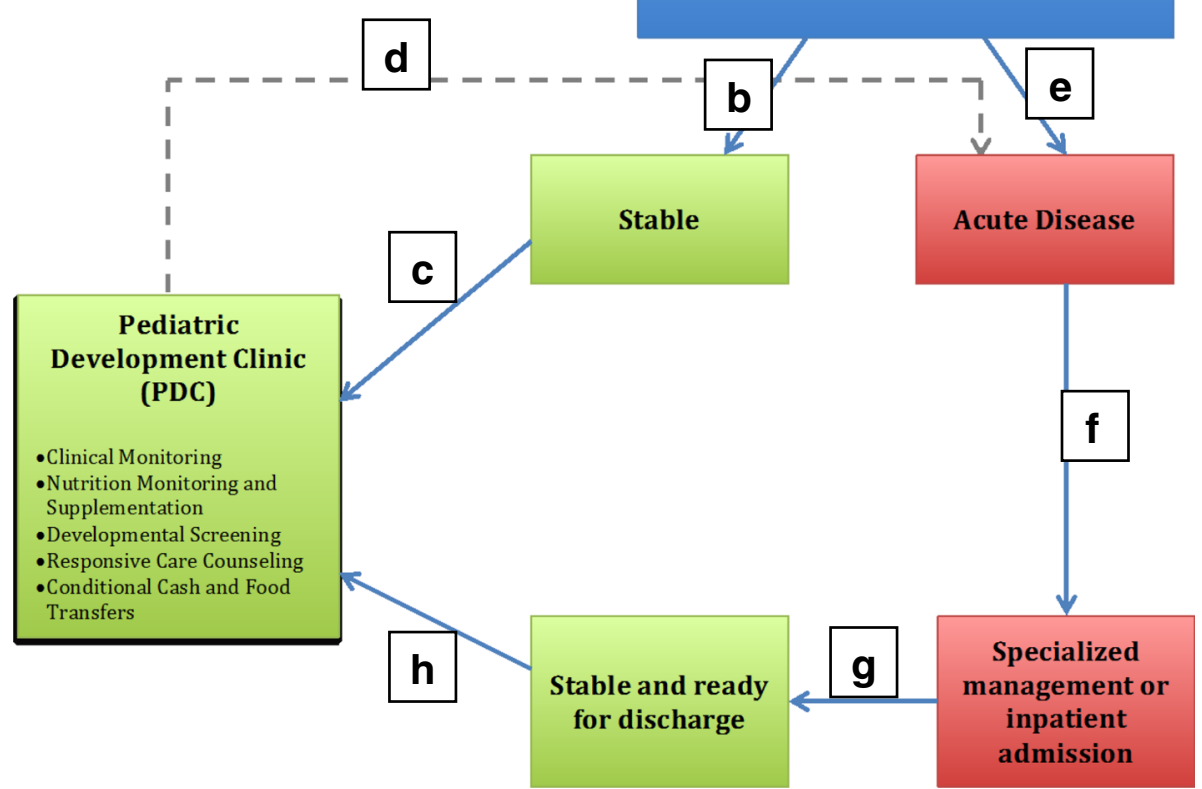

Fig. 1 Pediatric Development Clinic (PDC) Referral and Clinic Flow. Routine Clinic Flow: Different health facilities and services (a) refer all stable underfive children meeting referral criteria (b) to PDC for enrolment (c). At any PDC visit assessment, if an acute illness is identified, an immediately referral (d) for acute care is issued (e) followed by specialized care (f) if needed. When the child has been stabilized and preparing for hospital discharge ( $\mathbf{g}$ ), a referral back to continue PDC follow-up (h) is completed 
criteria is identified. The goal is to identify at-risk infants as young as possible for early enrollment. Children may additionally be referred informally from outpatient clinics at health centers and Rwinkwavu District Hospital, health center maternity wards or via self-referral from the community.

The clinic was designed to be integrated into the public system with as minimal additional external resources as possible to maximize the potential for scalability. Building on an existing platform for chronic care services, the PDCs were integrated into the noncommunicable disease (NCD) clinics in the public health system. Only one additional staff member was hired at the district hospital clinic due to the addition of patients to an already high-volume NCD clinic. The PDC is operated at each site on a weekly basis. The PDC team is led by a general practitioner based at the Rwinkwavu District Hospital PDC with routine clinic visits conducted by a nurse and social worker. Each clinic is equipped with a child-friendly space for group counseling and play, and a consultation room with basic furnishings, medical equipment for assessing growth and vitals, as well as structured medical record forms for monitoring patients. Health center teams receive mentorship and supervision from the district hospital team, and the hospital team receives mentorship from additional technical advisors as needed (including a pediatrician and a nutritionist). Prior to initiation of PDC, each team was trained on the PDC protocol and completion of medical record forms for each specific condition. When a health center clinic is first opened, the district hospital doctor, nurse and social worker provide weekly mentorship to provide intensive support following the initial training. After approximately two to 3 months of weekly mentorship (and depending on assessed level of skill and comfort of the providers) mentorship de-intensifies to at least one visit per month. The supervision team meets monthly to discuss strengths and weaknesses of implementation at each clinic, address specific challenges, and create future plans for the clinic.

On a visit day, children's clinical, nutritional and developmental status are assessed by the nurse. According to the protocol, the following are documented for all children: age, weight, height, interval growth for infants up to 6 months, head circumference for infants up to 18 months, immunization status, and vital signs. The child's anthropometric measurements are plotted on World Health Organization growth charts $[25,26]$ and counseling provided accordingly. Also, a conditionspecific protocol of additional screening is completed based on the reason for referral. For preterm infants the primary referral base - this includes Kangaroo Mother Care (KMC) follow-up, micronutrient supplementation, and vision/hearing screening among other standard follow-up measures. Developmental screening using an Ages and Stages Questionnaire (ASQ-3) [27] is conducted at ages $6,12,24,36,48$ and 60 months; the ASQ-3 was previously adapted and translated into the local language, Kinyarwanda, for use in Rwanda [19].

In addition, the children's primary caretakers participate in group education led by the social worker on different topics depending on the age of their children such as KMC, hygiene, nutrition, family planning, breastfeeding, and the importance of play and early stimulation. A structured social assessment is performed for further individual counseling and to identify families who qualify for conditional food or cash transfers, or additional counseling and home visits according to pre-determined clinical and social criteria. Rwanda's community-based poverty categorizations, known as Ubudehe, were used to establish social need [28]; Ubudehe is a government mechanism to help communities identify the most vulnerable households so that government-provided social support to achieve poverty reduction can be targeted to these households. Home visits are conducted weekly by social workers with nurses to provide support to families identified during clinic and social assessments as needing additional or home-based support.

\section{Data collection}

Data were extracted from electronic medical records (EMR) and paper-based patient charts by trained data collectors. The EMR undergoes routine data quality checks, and discrepancies for key indicators between paper-based charts and the EMR were addressed during data collection.

\section{Measures}

Demographic characteristics for children and their primary caretakers were extracted from patients' referral and intake forms. Children's age was categorized as neonates less than 1 month, infants 1 to 5 months, 6 to 11 months and children 12 months and above. Gestational age in weeks was categorized according to WHO classification as "extremely preterm" or less than 28 weeks, "very preterm" from 28 to less than 32 weeks, "moderate to late preterm" from 32 to less than 37 weeks and "term" as 37 weeks and above. Birth weight in grams was categorized as "extremely low birth weight" (less than $1000 \mathrm{~g}$ ), "very low birth weight" (from 1000 to 1499 g), "low birth weight" (1500 to $1999 \mathrm{~g}$ ) and birth weight above $2000 \mathrm{~g}$. Mother's age was categorized in intervals from 15 to less than 20, from 20 to 24,25 to 34 and 35 to 44 years and 45 years and above. Caretakers' socio-economic status was categorized using Rwanda's Ubudehe classification based on households that qualify for automatic government support or those who do not qualify. The number of other dependents in a household 
was categorized as "none" if the child attending PDC was the only child in the home, or " $1-3$ ", " 4 to 5 ", or " 6 and above".

Based on visit forms that were completed at each patient visit, we assessed whether the nurse had conducted the required assessments at each patient visit according to the PDC protocol. To assess changes in clinic implementation over time, patient visits were grouped into 6 months intervals: April to Sept 2014, October 2014 to March 2015, April to September 2015 and October 2015 to March 2016.

\section{Analysis}

We described patient demographic and clinical characteristics, and programmatic process using frequencies and proportions for categorical variables. We compared percent of different aspects of PDC protocol completed at each visit across 6 months intervals using a nonparametric test for trend [29] to determine if there were significant differences in protocol adherence over time.

\section{Ethics}

The Rwanda National Ethics Committee (RNEC) No.882/RNEC/2016 and Ministry of Health approved this study. Additionally, the institutional review board at Boston Children's Hospital (Boston, Massachusetts) exempted this study from review.

\section{Results}

From April 2014 to December 2015, 426 children enrolled in the PDC. Table 1 describes characteristics of the participants and their caregivers. Of 417 children with gender recorded, more than half were female $(54.2 \%, n=225 / 417)$. Most were neonates $(43.7 \%$, $n=172 / 394)$ or less than 6 months old (35.0\%, $n=138$ / 394 ) at the time of enrollment. Most enrollees $(56.7 \%$, $n=160 / 282$ ) were preterm. The clinic also enrolled children with a birth weight under $2000 \mathrm{~g}$, with 53.5\% ( $n=124 / 334)$ of those with available birth weight data in the low, very low or extremely low birthweight categories. Most enrolled children were born at Rwinkwavu District Hospital $(64.7 \%, n=247 / 382)$ and were referred into PDC from hospital departments. Two-thirds of children $(64.3 \%, n=214 / 333)$ were living in households with one to three other dependents.

Almost all children were brought to the PDC by their mothers $(98.1 \%, n=306 / 312)$. The majority of caregivers were between 25 and 34 years of age $(41.1 \%, n=120$ / $292)$, and were married or cohabitating $(88 \%, n=337$ / $383)$, and had some primary school education $(41.4 \%$, $n=142 / 343)$. Nearly all patients were living in Rwinkwavu catchment area $(92.7 \%, n=383 / 413)$ with some patients coming from neighboring hospital catchment areas. Of caregivers, $6.3 \%(n=23 / 367)$ were known to be living with HIV. The most common mode of transport to the clinic was walking (43.4\%, $n=177$ / $408)$ or motorcycle taxis $(36.5 \%, n=149 / 408)$. Of those with Ubudehe status recorded, 71.4\% ( $n=202 / 282)$ percent did not qualify for automatic government support.

The most frequent diagnosis of PDC clinic patients was prematurity and/or low birth weight (62.9\%, $n=266 / 423)$ followed by HIE $(33.7 \%, n=142 / 422$, Table 2). Less common diagnoses included hydrocephalus $(1.3 \%, n=5 / 390)$, trisomy $21(2.3 \%, n=9 / 390)$, and cleft lip and/or palate $(1.8 \%, n=7 / 390)$. Fifty-six children had more than one diagnosis (13.2\%).

We assessed whether families had received social support and developmental screening with the ASQ-3 according to protocol (Table 3). Of 364 participants with social support data available, almost every mother received transport fees at one or more visits during PDC $(96.7 \%, n=352)$ and $43.7 \%$ received food packages $(n=159)$. Additionally, more than one-third of children with available data $(36.3 \%, n=132)$ received infant formula for some period of time as part of a treatment plan for insufficient maternal breastmilk supply or other difficulties with breastfeeding to achieve adequate growth. Developmental screening using the ASQ-3 was completed for more than the half of children in the clinic who were 6 months or older $(56.4 \%, n=184 / 326$ eligible children 6 months or older).

The proportion of visits over time with clinical, nutritional and developmental screening and counseling completed according to protocol are shown in Table 4 . Overall, age, weight, height/length, and head circumference were measured consistently at visits (98.3\%, $n=2486 / 2529 ; 98.9 \%, n=2755 / 2787 ; 98.2 \%, n=2736 /$ 2787 and 95.8\%, $n=2366 / 2431$ respectively). Vaccination status was assessed at $88.1 \%(n=2138 / 2529)$ of visits and feeding difficulties were assessed at $84.5 \%$ $(n=2138 / 2529)$ of visits. Danger signs were assessed at $70.1 \%(n=1953 / 2787)$ of visits and interval growth for children under 6 months was assessed at 70.4\% ( $n=1168 / 2431)$ of visits. Child development activities were assessed less frequently; general child development counseling was provided $52.1 \%$ of the time and availability of playthings for stimulation in the home was assessed at $6.6 \%$ of visits. Adjusted age for preterm infants was assessed $21.6 \%(n=232 / 1074)$ of the time for those children under age 24 months with a documented gestational age recorded.

Significant, steady improvements in assessment over time were documented in development counseling from $21.8 \%(n=64 / 2675)$ in the first 6 months to $65.6 \%$ $(n=554 / 2675)$ in the most recent 6 months of clinic implementation $(p<0.001)$ as well as evaluation of home stimulation activities from $2.5 \%(n=7 / 2529)$ to $8.5 \%$ ( $n=69 / 2529, p=0.001)$. Danger signs assessment 
Table 1 Demographic characteristics of children enrolled in PDCs from April 2014 to March 2016 and their caretakers

\begin{tabular}{|c|c|c|c|c|c|}
\hline \multirow[t]{2}{*}{ Child characteristics } & \multicolumn{2}{|c|}{ Total $N=426$} & \multicolumn{3}{|c|}{ (Continued) } \\
\hline & $n$ & $\%$ & HIV status $(N=367)$ & & \\
\hline Gender $(N=415)$ & & & Positive & 23 & 6.3 \\
\hline Male & 190 & 45.8 & Negative & 344 & 93.7 \\
\hline Female & 225 & 54.2 & Caretaker education level $(N=343)$ & & \\
\hline Age at first visit $(N=394)$ & & & No formal education & 57 & 16.6 \\
\hline Neonate (< 1 month) & 172 & 43.7 & Some primary & 142 & 41.4 \\
\hline $1-5$ months & 138 & 35.0 & Primary completed & 128 & 37.3 \\
\hline 6-11 months & 26 & 6.6 & Secondary or higher completed & 16 & 4.7 \\
\hline 12 months and above & 58 & 14.7 & Ubudehe classification ( $N=282$ ) & & \\
\hline Gestational age in weeks $(N=282)$ & & & Does not qualify for government support & 202 & 71.4 \\
\hline Extremely preterm ( $<28$ weeks) & 13 & 4.6 & Qualifies for government support & 80 & 28.3 \\
\hline Very preterm (29 to <32 weeks) & 38 & 13.5 & Household lives within Rwinkwavu catchment ar & $=413)$ & \\
\hline Moderate to late preterm (32 to <37 weeks) & 109 & 38.7 & Yes & 383 & 92.7 \\
\hline Term (37 + weeks) & 122 & 43.3 & No & 30 & 7.3 \\
\hline Birth weight categories (grams) $(N=334)$ & & & Transport means to the health facility $(N=408)$ & & \\
\hline Extremely low, < $1000 \mathrm{~g}$ & 4 & 1.2 & Walking & 177 & 43.4 \\
\hline Very low, 1000-1499 g & 61 & 18.3 & Bicycle & 4 & 1.0 \\
\hline Low, 1500-1999 g & 114 & 34.1 & Motorbike & 149 & 36.5 \\
\hline $2000 \mathrm{~g}$ or more & 155 & 46.4 & Mini bus & 54 & 13.2 \\
\hline Site of birth $(N=382)$ & & & Other & 24 & 5.9 \\
\hline District Hospital & 247 & 64.7 & Number of dependents in a household $(N=333$ & & \\
\hline Health Center & 97 & 25.4 & No other children & 23 & 6.9 \\
\hline Home & 26 & 6.8 & 1 to 3 & 214 & 64.3 \\
\hline En route & 8 & 2.1 & 4 to 5 & 66 & 19.8 \\
\hline National Referral Hospital & 4 & 1.1 & 6 and above & 30 & 9.0 \\
\hline
\end{tabular}

Table 1 Demographic characteristics of children enrolled in PDCs from April 2014 to March 2016 and their caretakers Continued)

improved substantially between the first and second 6 months from $43.2 \%(n=136 / 2787)$ to $72.2 \%(n=625 /$ $2787)$, then stabilized $(p<0.001)$. Feeding difficulty and vaccination status assessments were more varied across time: feeding difficulty fluctuated from $88.1 \%(n=244$ / $2529)$ in the first 6 months to $78.6 \%(n=496 / 2529)$ then $82.2 \%(n=664 / 2529)$ and up to $90.3 \%(n=734 / 2529)$ in the most recent 6 months of implementation $(p<0.001)$. Vaccination status also varied, with some declines from $93.5 \%(n=259 / 2529)$ in the first 6 months of implementation to $87.6 \%(n=553 / 2529)$, then $86.1 \%$ $(n=696 / 2529)$ and $88.7 \%(n=721 / 2529, p=0.171)$. Proportion of interval growth assessment dropped significantly in the last 6 months from $74.5 \%(n=196 /$ $1659)$ to $57.3 \%(n=236 / 1659, p<0.001)$.

\section{Discussion}

We report the successful implementation of the novel PDC in a rural African setting during its first 24 months of operation. Our findings show relative completeness of follow-up visits according to PDC protocol for 2 years in 
Table 2 Medical diagnoses of PDC patients at enrollment in Rwinkwavu catchment area

\begin{tabular}{lll}
\hline Medical conditions & $\mathrm{n}$ & $\%$ \\
\hline Children with any diagnosis on record & & \\
Diagnosed as preterm/LBW $(N=423)$ & 266 & 62.9 \\
Diagnosed with HIE $(N=422)$ & 142 & 33.7 \\
Diagnosed with Hydrocephalus $(N=390)$ & 5 & 1.3 \\
Diagnosed with Trisomy 21 ( $=390)$ & 9 & 2.3 \\
Diagnosed with Cleft lip and/or palate $(N=390)$ & 7 & 1.8 \\
Diagnosed with other development delays $(N=390)$ & 22 & 5.6 \\
Diagnosed with other condition $(N=390)$ & 22 & 5.6 \\
Child has multiple diagnoses $(N=423)$ & 56 & 13.2 \\
\hline
\end{tabular}

this population of previously underserved high-risk children. In a setting with very few specialists, the program was designed to tackle a health worker shortage based on a task shifting model that relies primarily on nurses and social workers to deliver care with general practitioner oversight. Our findings show this task shifted model is feasible for delivering follow-up care for the early identification of complications among a population of children at risk of medical, nutritional, and developmental impairments. This builds on the large body of evidence demonstrating successes of task shifting in other domains, especially in HIV care [30].

The PDC is reaching a diverse population of at-risk children under- 5 years of age, many of whom were previously not reached at all [31]. By providing services for these high-risk children, PDC has the potential to empower caretakers who face stigma and high levels of stress when looking after children experiencing developmental problems [32]. The targeted population of PDC was focused on those infants born with perinatal risk whose long-term outcomes could benefit significantly with early assessment and intervention to catch up with their non-medically vulnerable peers- namely, those born preterm and/or low birth weight and significant HIE, as well as other less common perinatal conditions requiring medical and nutritional follow-up. The

Table 3 Developmental screening and social support provided to PDC patients at enrolment

\begin{tabular}{|c|c|c|}
\hline & $\mathrm{n}$ & $\%$ \\
\hline \multicolumn{3}{|c|}{ Socioeconomic assistance ever received $(N=364)$} \\
\hline Transport fees & 352 & 96.7 \\
\hline Infant formula & 132 & 36.3 \\
\hline Food package & 159 & 43.7 \\
\hline \multicolumn{3}{|c|}{ ASQ assessment completed if child is $6+$ months old $(N=326)$} \\
\hline Yes & 184 & 56.4 \\
\hline No & 142 & 43.6 \\
\hline
\end{tabular}

majority of children were less than 6 months at the time of enrollment, which is critical for ensuring services are provided as early as possible. By enrolling children in their first few months of life and routinely screening these children for medical, nutritional and developmental concerns, the PDC has created a platform for early identification and intervention rather than the default system of waiting until children are sick and present to care before care is provided [33]. Additionally, creating a comprehensive platform rather than a vertical program targeted solely at one population, such as preterm infants for $\mathrm{KMC}$, optimizes the impact of investing in a chronic care operational delivery model.

Most caretakers were mothers and were married or cohabiting. Caretakers play an enormous role in the development of young children and the very rare involvement of father's in the program is an aspect that the clinic is trying to address. Involving fathers in children's care can mean not only that when mothers are not available, they can step in and avoid missing appointments, but more importantly, that fathers are also involved in early stimulation activities at home [34] which can improve children's developmental outcome [35]. Additionally, supporting older siblings who may serve as caretakers is an area for consideration in future programming. Further, almost half of caretakers were less than 25 years of age, with several adolescent mothers, which has been shown to be associated with social and medical vulnerability and a barrier to adherence to care [36]. This unique population of mothers can benefit from services promoting positive parenting and stimulation [37] to empower them to create a nurturing home environment for optimal development.

In serving this unique and vulnerable population of patients, the provision of conditional food and cash transfers aims to break barriers to adherence to chronic care and ensure adequacy of access to at least minimum nutritional needs. As the PDC patients' families were primarily not eligible for government support, social support is an integral component of the PDC program. The majority of patients in the program benefit from some form of conditional food and cash transfers, particularly transport fees to reduce financial barriers to accessing the PDC. Future research to assess PDC impact on patient outcomes is needed, which, in combination with a costing analysis - that takes into consideration required start-up costs for equipment and staff training, staff time, social supports, medications, supplies, operational costs, as well as costs of mentorship and supervision for both health center and hospital teams - will be important to assessing potential for scale-up.

Although there was significant variability in the proportion of visit assessments completed over time, 
Table 4 Progress of Pediatric Development Clinics services in the first 24 months of implementation

\begin{tabular}{|c|c|c|c|c|c|c|c|c|c|}
\hline \multirow[t]{2}{*}{ Time periods } & \multicolumn{2}{|c|}{ 0-6 months } & \multicolumn{2}{|c|}{$7-12$ months } & \multicolumn{2}{|c|}{ 13-18 months } & \multicolumn{2}{|c|}{ 19-24 months } & \multirow[b]{2}{*}{$P$ value* } \\
\hline & $\bar{n}$ & $\%$ & $\mathrm{n}$ & $\%$ & n & $\%$ & n & $\%$ & \\
\hline Total number of visits during period & 315 & 11.3 & 716 & 25.7 & 890 & 31.9 & 866 & 31.1 & \\
\hline \multicolumn{10}{|l|}{ Visit location $(N=2787)$} \\
\hline Rwinkwavu District Hospital & 250 & 79.4 & 363 & 50.7 & 391 & 43.9 & 444 & 51.3 & $<0.001$ \\
\hline Decentralized Health Center & 65 & 20.6 & 353 & 49.3 & 499 & 56.1 & 422 & 48.7 & \\
\hline \multicolumn{10}{|l|}{ Danger signs assessed at visit $(N=2787)$} \\
\hline Yes & 136 & 43.2 & 508 & 71.0 & 684 & 76.9 & 625 & 72.2 & $<0.001$ \\
\hline No & 179 & 56.8 & 208 & 29.1 & 206 & 23.2 & 241 & 27.8 & \\
\hline \multicolumn{10}{|l|}{ Age recorded at visit $(N=2529)$} \\
\hline Yes & 272 & 98.2 & 617 & 97.8 & 795 & 98.4 & 802 & 98.7 & 0.302 \\
\hline No & 5 & 1.8 & 14 & 2.2 & 13 & 1.6 & 11 & 1.4 & \\
\hline \multicolumn{10}{|l|}{$\begin{array}{l}\text { Adjusted age for preterm babies } \\
\leq 24 \text { months }(N=1074)\end{array}$} \\
\hline Yes & 27 & 20.8 & 91 & 31.1 & 64 & 18.2 & 50 & 16.7 & 0.003 \\
\hline No & 103 & 79.2 & 202 & 68.9 & 288 & 81.8 & 249 & 83.3 & \\
\hline \multicolumn{10}{|l|}{ Weight recorded at visit $(N=2787)$} \\
\hline Yes & 312 & 99.1 & 708 & 98.9 & 876 & 98.4 & 859 & 99.2 & 0.790 \\
\hline No & 3 & 1.0 & 8 & 1.1 & 14 & 1.6 & 7 & 0.8 & \\
\hline \multicolumn{10}{|l|}{ Height recorded at visit $(N=2787)$} \\
\hline Yes & 308 & 97.8 & 695 & 97.1 & 877 & 98.5 & 856 & 98.9 & 0.021 \\
\hline No & 7 & 2.2 & 21 & 2.9 & 13 & 1.5 & 10 & 1.2 & \\
\hline \multicolumn{10}{|l|}{$\begin{array}{l}\text { Head circumference recorded at visit if } \\
\leq 18 \text { months }(N=2431)\end{array}$} \\
\hline Yes & 284 & 96.3 & 607 & 92.1 & 779 & 97.4 & 696 & 97.3 & $<0.001$ \\
\hline No & 11 & 3.7 & 52 & 7.9 & 21 & 2.6 & 19 & 2.7 & \\
\hline \multicolumn{10}{|l|}{$\begin{array}{l}\text { Interval growth calculated at visit if } \\
\leq 6 \text { months }(N=1659)\end{array}$} \\
\hline Yes & 196 & 74.5 & 385 & 76.4 & 351 & 73.1 & 236 & 57.3 & $<0.001$ \\
\hline No & 67 & 25.5 & 119 & 23.6 & 129 & 26.9 & 176 & 42.7 & \\
\hline \multicolumn{10}{|l|}{ Vaccination status recorded $(N=2529)$} \\
\hline Yes & 259 & 93.5 & 553 & 87.6 & 696 & 86.1 & 721 & 88.7 & 0.171 \\
\hline No & 18 & 6.5 & 78 & 12.4 & 112 & 13.9 & 92 & 11.3 & \\
\hline \multicolumn{10}{|l|}{ Feeding difficulty assessed $(N=2529)$} \\
\hline Yes & 244 & 88.1 & 496 & 78.6 & 664 & 82.2 & 734 & 90.3 & $<0.001$ \\
\hline No & 33 & 11.9 & 135 & 21.4 & 144 & 17.8 & 79 & 9.7 & \\
\hline \multicolumn{10}{|l|}{$\begin{array}{l}\text { General child development counseling } \\
\text { received }(N=2675)\end{array}$} \\
\hline Yes & 64 & 21.8 & 256 & 38.2 & 519 & 59.9 & 554 & 65.6 & $<0.001$ \\
\hline No & 229 & 78.2 & 415 & 61.9 & 347 & 40.1 & 291 & 34.4 & \\
\hline \multicolumn{10}{|l|}{$\begin{array}{l}\text { Playthings and stimulating activities in } \\
\text { the home assessed }(N=2529)\end{array}$} \\
\hline Yes & 7 & 2.5 & 32 & 5.1 & 58 & 7.2 & 69 & 8.5 & $<0.001$ \\
\hline No & 270 & 97.5 & 599 & 94.9 & 750 & 92.8 & 744 & 91.5 & \\
\hline \multicolumn{10}{|l|}{ Next rendez-vous recorded $(N=2787$ ) } \\
\hline Yes & 309 & 98.1 & 694 & 96.9 & 869 & 97.6 & 841 & 97.1 & 0.658 \\
\hline No & 6 & 1.9 & 22 & 3.1 & 21 & 2.4 & 25 & 2.9 & \\
\hline
\end{tabular}


completion of screening for danger signs and developmental counselling improved significantly. Possible explanations for this finding are that over time, providers became more familiar and more comfortable with assessing these components. Additionally, mentorship visits were conducted at least monthly by the hospital staff to health center clinics where the district hospital PDC team provided practice-based guidance on care protocols, referral processes, and nutrition and development counseling to health center teams. These visits have likely contributed to this improvement. Percent complete recording of adjusted age, although improved since the first 6 months of PDC, have fluctuated over time. Interval growth calculation was steadily above $70 \%$ until the last 6 months, when we saw a sharp reduction in percent complete recording. These quality gaps highlight the particular challenges of growth monitoring in preterm infants where growth standards are unique and gestational age is often not known. We found that variables requiring calculation, such as adjusted age for preterm infants and interval growth calculation, were completed less frequently than those variables requiring simple recording, such as age, weight and head circumference. These well-done components are also captured in other clinics, such as the Integrated Management of Childhood Illness, and clinicians are accustomed to recording them. Although a clinician's ability to calculate interval growth will be impacted if the weight of the previous visit was not recorded in patient charts, the fact that weight was recorded more than $95 \%$ of the time makes this less likely to be an issue. Qualitative exploration of factors leading to the reduction in recording are needed to understand these findings. Automated calculation tools, such as job aids or mHealth tools, could facilitate the completion of these assessments, provide clinical reminders, and video teaching and counseling supports, as has been successfully done in other taskshifted programs [38, 39].

More than the half of PDC children who were eligible for ASQ had one completed. The integration of developmental screening has proven challenging as nurses have to read questions to caretakers due to low literacy and it takes time to administer, which can be particularly burdensome as patient volume increases. Furthermore, the ASQ-3 was a new tool in our setting and nurses from PDCs needed time to become familiar with the process. Further efforts are needed to be able to appropriately translate the findings of a developmental screening into direct intervention in settings such as Rwanda where no locally validated tools exist [18]. Some promising tools have emerged that were designed specifically for easy assessment and intervention in low- and middle-income countries including the International Guide for Monitoring Child Development [40] and the Caregiver-Reported
Early Childhood Development Index (CREDI) [41], which could be more appropriate but require additional study for feasibility in the PDC. In the meantime, a play and communication module has been introduced more recently into the protocol and provider training in order to help boost the developmental assessment and intervention skills of PDC providers.

This study has some limitations. Because we used routinely-collected data from patient charts entered into EMR, data were missing or incomplete for several variables. However, we believe the data available gives an accurate description of the clinic's patients and services. In addition, when possible, we verified discrepant data in patient charts and in the EMR. Another limitation could be the generalizability of the study findings to other settings, as the pilot for PDC was conducted in rural southern Kayonza District in Rwinkwavu District Hospital catchment area which has received support from Partners In Health/Inshuti Mu Buzima since 2005.

\section{Conclusion}

Our study demonstrates that the PDC model is a feasible design that supports early identification and intervention for medical, nutritional, and developmental complications among high-risk children in a rural African setting. Using a task-shifting model, nurses and social workers were able to follow a standardized protocol for patient visits; however as a new program there are areas for ongoing improvement in the quality of care. Future research is needed to understand the outcomes of the children benefiting from the PDC program as well as costs of PDC implementation.

\section{Acknowledgements}

We acknowledge Partners In Health/Inshuti Mu Buzima for the support of this work. This study was developed under the Partners In Health/Inshuti Mu Buzima Intermediate Operational Research Training Program, developed and facilitated by Bethany Hedt-Gauthier and Jackline Odhiambo. Ann Miller and Catherine Kirk provided direct mentorship to this paper as part of this training. Training costs were covered by the Harvard Global Health Initiative Burke Global Health Fellowship grant and by Partners In Health/Inshuti Mu Buzima. Some training facilitators and mentors are Rwanda Human Resources for Health faculty, funded with the support of the Government of Rwanda, the Global Fund to Fight AIDS, Tuberculosis and Malaria and Centers for Disease Control and Prevention. Support for some mentors was provided by the Global Health Research Core at Harvard Medical School. We also acknowledge the contributions of Stephanie Bazubagira, Jean de Dieu Uwihaye, and Emmanuel Ndayishimiye for data collection as well as Kaya Hedt for her support of data cleaning

\section{Funding}

This manuscript was developed under the Partners In Health/Inshuti Mu Buzima Intermediate Operational Research Training Program, training costs were covered by the Harvard Global Health Initiative Burke Global Health Fellowship grant and by Partners In Health/Inshuti Mu Buzima. Some training facilitators and mentors are Rwanda Human Resources for Health faculty, funded with the support of the Government of Rwanda, the Global Fund to fight AIDS, Tuberculosis and Malaria and Centers for Disease Control and Prevention. Support for some mentors was provided by the Global Health Research Core at Harvard Medical School. Data collection costs were covered by UNICEF Rwanda. 


\section{Availability of data and materials}

The data that support the findings of this study are available from the Rwandan Ministry of Health but restrictions apply to the availability of these data which were used under license for the current study, and are not publicly available. Data are however available from the authors upon reasonable request and with the permission of $\mathrm{MOH}$.

\section{Authors' contributions}

CM \& EN designed the protocol, analyzed the data, and wrote the first and subsequent drafts of the manuscript. HM \& KW designed the PDC program, assisted with protocol design, analysis contextualization, and reviewed all drafts of the manuscript. AM \& CK mentored the protocol design, data analysis and drafts of the paper, and reviewed all versions of the manuscript. CK also cleaned the data. ED and FN reviewed the manuscript, OB and SE work on PDC implementation directly and reviewed the manuscript. Al authors read and approved the final manuscript.

\section{Competing interests}

The authors declare that they have no competing interests.

\section{Consent for publication}

Not applicable.

\section{Ethics approval and consent to participate}

The Rwanda National Ethics Committee (RNEC) No.882/RNEC/2016 and Ministry of Health approved this study. Additionally, the institutional review board at Boston Children's Hospital (Boston, Massachusetts) exempted this study from review.

\section{Publisher's Note}

Springer Nature remains neutral with regard to jurisdictional claims in published maps and institutional affiliations.

\section{Author details}

'Rwinkwavu District Hospital, Ministry of Health, Rwinkwavu, Rwanda. 2Department of Pediatrics, Partners In Health/Inshuti Mu Buzima, Rwinkwavu, Rwanda. ${ }^{3}$ Division of Global Health Equity, Brigham and Women's Hospital, Boston, USA. ${ }^{4}$ Division of General Pediatrics, Boston Children's Hospital, Boston, USA. ${ }^{5}$ Harvard Medical School, Department of Global Health and Social Medicine, Boston, USA. 'Division of Clinical Services, Ministry of Health, Kigali, Rwanda. ${ }^{7}$ P.O. Box 3432, Kigali, Rwanda.

Received: 8 March 2017 Accepted: 7 June 2017

\section{Published online: 12 July 2017}

\section{References}

1. Darmstadt GL, Shiffman J, Lawn JE. Advancing the newborn and stillbirth global agenda: priorities for the next decade. Arch Dis Child. 2015;100(Suppl 1):S13-8.

2. Lu C, Black MM, Richter L. Risk of poor development in young children in low-income and middle-income countries: an estimation and analysis at the global, regional, and country level. Lancet Glob Health. 2016;4(12):e916-e22.

3. Lawn JE, Blencowe H, Oza S, You D, Lee AC, Waiswa P, et al. Every Newborn: progress, priorities, and potential beyond survival. Lancet. 2014; 384(9938):189-205.

4. Gilmore JH, Lin W, Prastawa MW, Looney CB, Vesta YS, Knickmeyer RC, et al. Regional gray matter growth, sexual dimorphism, and cerebral asymmetry in the neonatal brain. J Neurosci. 2007;27(6):1255-60.

5. Ford ND, Stein AD. Risk factors affecting child cognitive development: a summary of nutrition, environment, and maternal child interaction indicators for Sub- Saharan Africa. J Dev Orig Health Dis. 2016;7(2):197-217.

6. Casale D, Desmond C, Richert L. The association between stunting and psychosocial development among preschool children: a study using the South African Birth to Twenty cohort data. Child Care Health Dev. 2014; 40(6):900-10.

7. Aarnoudse-Moens CS, Weisglas-Kuperus N, Van Goudoever JB, Oosterlaan J. Meta- Analysis of Neurobehavioral Outcomes in Very Preterm and/ or Very Low Birth Weight Children. Pediatrics. 2009;124(2):717-28.

8. Colella-Santos MF, Hein TA, de Souza GL, do Amaral MI, Casali RL. Newborn Hearing Screening and Early Diagnostic in the NICU. Biomed Res Int. 2014; 2014:845308. doi:10.1155/2014/845308.
9. Zerbeto AB, Cortelo FM, Filho EB C. Association between gestational age and birth weight on the language development of Brazilian children: a systematic review. J Pediatr. 2015;91(4):326-32.

10. Ballot DE, Potterton J, Chirwa T, Hilburn N, Cooper PA. Developmental outcome of very low birth weight infants in a developing country. BMC Pediatr. 2012:12:11. doi:10.1186/1471-2431-12-11.

11. Sudfeld CR, McCoy DC, Fink G, Muhihi A, Bellinger DC, Masanja H, et al. Malnutrition and Its Determinants Are Associated with Suboptimal Cognitive, Communication and Motor Development in Tanzanian Children. J Nutr. 2015;145(12):2705-14.

12. Doyle LW, Anderson PJ, Battin M, Bowen JR, Brown N, Callanan C, et al. Long term follow up of high risk children: who, why and how? BMC Pediatr. 2014;14:279. doi:10.1186/1471-2431-14-279.

13. Huang KY, Nakigudde J, Calzada E, Boivin MJ, Ogedegbe G, Brotman LM. Implementing an early childhood school- based mental health promotion intervention in low-resource Ugandan Schools: study protocol for a cluster randomized controlled trial. Trails. 2014:15(1):471. doi:10.1186/ 1745-6215-15-471.

14. Yousfazai AK, Rasheed MA, Rizvi A, Amstrong R, Bhutta ZA. Parenting Skills and Emotional Availablity: An RCT. Pediatrics. 2015;135(5):e1247-57.

15. Wallender JL, Bann CM, Biasini FJ, Goudar SS, Pasha O, Chomba E, et al. Development of children at risk for adverse outcomes participating in early intervention in developing countries: a randomized controlled trial. J Child Psychol Psychiarty. 2014;55(11):1251-9.

16. Farmer PE, Nutt $C T$, Wagner $C M$, Sekabaraga $C$, Nuthulaganti $T$, Weigel JL, et al. Reduced premature mortality in Rwanda: lessons from success. BMJ. 2013;346:f65.

17. Katz J, Lee AC, Kozuki N, Lawn JE, Cousens S, Blencowe H, et al. Mortality risk in preterm and small-for-gestational-age infants in low-income and middle-income countries: a pooled country analysis. Lancet. 2013;382(9890): $417-25$

18. Rwanda Ministry of Health. National Community Health Policy. 2015. http:// www.moh.gov.rw/fileadmin/templates/policies/NATIONAL_COMMUNITY_ HEALTH_POLICY.pdf. 24 Jan 2017.

19. UNICEF. Early Childhood Development and Family Services: Baseline Evaluation in 2016 Sites in Rwanda. https://www.unicef.org/evaldatabase/ files/ECD_and_F_Baseline_Evaluation_Rwanda.pdf. 12 Dec 2016.

20. USAID. An Evaluation of the Kuraneza Project: Good Growth for Children in Rwanda. 2015. http://www.mcsprogram.org/wp-content/uploads/2015/08/ CARE-Rwanda-FE-Brief1.pdf. Accessed 16 Nov 2016.

21. World Health Organization. Health workforce requirements for universal health coverage and the sustainable development goals. 2016. http://apps. who.int/iris/bitstream/10665/250330/1/9789241511407-eng.pdf?ua=1. Accessed 12 Jan 2016.

22. Shumbusho F, van Griensven J, Lowrance D, Turate I, Weaver MA, Price J, et al. Task shifting for scale-up of HIV Care: evaluation of nurse- centered antiretroviral treatment at rural health centers in Rwanda. PLoS Med. 2009; 6(10):e1000163.

23. National Institute of Statistics of Rwanda. Fourth Population and Housing Census, Rwanda, 2012: Final Results Main Indicators Report 2014. 16 Nov 2016.

24. Hansen A, Magge H, Labrecque M, Munyaneza RB, Nahimana E, Nyishime $\mathrm{M}$, et al. The development and implementation of a newborn medicine program in a resource-limited setting. Public Health Action. 2015;5(1):17-22.

25. World Health Organization. WHO Child Growth Standards: Length/heightfor-age, weight-for-age, weight-for-length, weight-for-height and body mass index-for-age: Methods and Development. Geneva: World Health Organization; 2006.

26. World Health Organization. WHO Child Growth Standards: Head Circumference-for-age, Arm Circumference-for- age, Triceps Skinfold-for-age and Subscapular Skinfold-for-age: Methods and Development. Geneva: World Health Organization; 2007.

27. Squires J, Twombly E, Bricker D, Potter L. ASQ-3 User's Guide. 2009. Baltimore: Paul H. Brookes Publishing Company. 2009.

28. Ministry of Finance and Economic Planning. The Government of Rwanda, Poverty Reduction Strategy Paper. 2002. http://www.unrwanda.org/undp/ prsp2002.pdf. Accessed 25 Jan 2017.

29. Cuzick J. A Wilcoxon-type test for trend. Stat Med. 1985:4:87-90.

30. World Health Organization. Task shifting to tackle health worker shortages. 2007. http://www.who.int/healthsystems/task_shifting_booklet.pdf. Accessed 11 Nov 2016. 
31. Blencowe H, Cousens S, Chou D, Oestergaard M, Say L, Moller AB, et al. Borrn To Soon: The global epidemiology of 15 million preterm births. Reprod Health. 2013;10(suppl 1):S2. doi:10.1186/1742-4755-10-S1-S2.

32. Masulani-Mwale $C$, Mathanga D, Silungwe $D$, Kauye F, Gladstone M. Parenting children with intellectual disabilities in Malawi: the impact that reaches beyond coping? Child Care. Health Dev. 2016;42(6):871-80.

33. Mugeni C, Levine AC, Munyaneza RM, Mulindahabi E, Crokrell HC, GlavisBloom J, et al. Nationwide implementation of integrated community case management of childhood illness in Rwanda. Glob Health Sci Pract. 2014; 2(3):328-41.

34. Britto PR, Lye SL, Proulx K, Yousfazai AK, Matthews SG, Vaivada T, et al. Nurturing care: promoting early childhood development. Lancet. 2017; 389(10064):91-102.

35. Jeong J, McCoy DC, Yousafzai AK, Salhi, C, Fink G. Paternal Stimulation and Early Child Development in Low- and Middle- Income Countries. Pediatrics. 2016;138(4):e20161357.

36. Pinto-Foltz MD, Logsdon C, Derrick A. Engaging adolescent mothers in a longitudinal mental health intervention study: challenges and lessons learned. Issues Ment Health Nurs. 2011;32(4):214-9.

37. Aboud FE. Evaluation of early childhood parenting program in rural Bangladesh. J Health Popul Nutr. J Health Popul Nutr. 2007;25(1):3-13.

38. Catalani C, Philbrick W, Fraser H, Mechael P, Israelski DM. mHealth for HIV treatment and Prevention: A systematic Review of the Literature. Open AIDS J. 2013;7:17-41.

39. Labrique AB, Vasudevan $L$, Kochi E, Fabricant $R$, Mehl G. mHealth innovations as health system strengthening tools: 12 common applications and a visual framework. Global Health: Science and Practice. 2013;1 (2):160-71.

40. Ertem IO, Dogan DG, Gok CG, Kizilates SU, Caliskan A, Atay G, et al. A Guide for Monitoring Child Development in Low and Middle-Income Countries. Pediatrics. 2008;121(3):e581-9.

41. McCoy DC, Sudfeld CR, Bellinger DC, Muhihi A, Ashery G, Weary TE et al. Development and validation of an early childhood development scale for use in low-resourced settings. Popul Health Metr. 2017;15(3). doi:10.1186/ s12963-017-0122-8.

\section{Submit your next manuscript to BioMed Central and we will help you at every step:}

- We accept pre-submission inquiries

- Our selector tool helps you to find the most relevant journal

- We provide round the clock customer support

- Convenient online submission

- Thorough peer review

- Inclusion in PubMed and all major indexing services

- Maximum visibility for your research

Submit your manuscript at www.biomedcentral.com/submit 